\title{
Xpp1 regulates the expression of xylanases, but not of cellulases in Trichoderma reesei
}

\author{
Christian Derntl ${ }^{1}$, Alice Rassinger ${ }^{1}$, Ewald Srebotnik ${ }^{2}$, Robert L Mach ${ }^{1}$ and Astrid R Mach-Aigner ${ }^{{ }^{*}}$
}

\begin{abstract}
Background: The ascomycete Trichoderma reesei is industrially used for the production of cellulases. During the production process xylanases are co-secreted, which uses energy and nutrients. Cellulases and xylanases share the same main regulators, which makes a knowledge-based strain design difficult. However, previously a cis-element in the promoter of the main xylanase-encoding gene was identified as binding site for a putative repressor. Subsequently, three candidate repressors were identified in a pull-down approach. The expression of the most promising candidate, Xpp1 (Xylanase promoter-binding protein 1), was reported to be up-regulated on the repressing carbon source D-glucose and to bind the cis-element in vitro.
\end{abstract}

Results: In this study, Xpp1 was deleted and over-expressed in T. reesei. An in vivo DNA-footprint assay indicated that Xpp1 binds a palindromic sequence in the xyn2 promoter. Comparison of the deletion, the over-expression, and the parent strain demonstrated that Xpp1 regulates gene expression of xylanolytic enzymes at later cultivation stages. Xpp1 expression was found to be up-regulated, additionally to D-glucose, by high D-xylose availability. These findings together with the observed $x y n 2$ transcript levels during growth on xylan suggest that Xpp1 is the mediator of a feedback mechanism. Notably, Xpp1 has neither influence on the D-xylose metabolism nor on the expression of cellulases.

Conclusions: Xpp1 as regulator acting on the expression of xylanases, but not cellulases, is a highly promising candidate for knowledge-based strain design to improve the cellulases-to-xylanases ratio during industrial cellulase production.

Keywords: Trichoderma reesei, Xylanases, Cellulases, Gene regulation, Transcription factor, Cellulosic ethanol

\section{Background}

The filamentous ascomycete Trichoderma reesei (teleomorph, Hypocrea jecorina) [1]) is industrially used for its outstanding secretory capacities. Industry strains produce over $100 \mathrm{~g} / \mathrm{L}$ of enzyme in industrial-scale processes [2]. The main component of the secreted enzymes is the cellobiohydrolase CBHI (EC 3.2.1.91). Further components of the secreted enzyme cocktail are the cellobiohydrolase CBHII (EC 3.2.1.91), the endoglucanase EGLI (EC 3.2.1.4), and the $\beta$-glucosidase BGLI (EC 3.2.1.21) $[3,4]$. These enzymes work synergistically to break down

\footnotetext{
*Correspondence: astrid.mach-aigner@tuwien.ac.at

${ }^{1}$ Department for Biotechnology and Microbiology, Institute of Chemical

Engineering, TU Wien, Gumpendorfer Str. 1a, 1060 Vienna, Austria

Full list of author information is available at the end of the article
}

cellulose to D-glucose. T. reesei cellulases find use in a wide range of industrial applications, such as paper and pulp, textile, and food and feed industry [5], and are still a bottleneck for cost-effective production of cellulosic ethanol [6].

However, T. reesei secretes also other enzymes along with the latter mentioned cellulases-most prominently the major endo- $\beta$-1,4-xylanase XYNII (EC.3.2.1.8) [7] and the $\beta$-xylosidase BXLI (EC 3.2.1.37) [8]. Endo- $\beta$-1,4xylanases cleave the backbone of xylan (i.e., the $\beta-1,4-$ $D$-xylose chain) in the middle, generating substrates for the $\beta$-xylosidase which cleaves off $\mathrm{D}$-xylose from the non-reducing ends of the xylan backbone. The cosecretion of cellulases and xylanases makes sense for the saprophyte $T$. reesei, because cellulose in plant material 
is encountered mostly exclusively together with hemicelluloses in the lignocellulose complex (reviewed in [9]). In industrial applications, however, the co-secretion of xylanases is a disadvantage for cost-efficient cellulase production because $T$. reesei uses additional energy and nutrients for their expression. Therefore, the reduction of xylanase formation during the cellulase production process is an obvious possibility for a more efficient cellulase production.

A way to achieve this would be knowledge-based strain design by deleting and/or over-expressing transcription factors that specifically regulate xylanase gene expression. However, the same main regulators regulate gene expression of cellulases and xylanases in $T$. reesei. The transactivator Xyr1 (Xylanase regulator 1) is essential for gene expression of most cellulolytic and xylanolytic enzymes [10]. Notably, cellulases seem to be regulated in a different manner by Xyr1 than xylanases. The up-regulation of xyr1 transcription goes hand in hand with the up-regulation of gene expression of cellulolytic enzymes, whereas the regulation of xylanases seems to depend on additional mechanisms and factors [11]. Further, gene expression of cellulases, xylanases, and their main regulator Xyr1 is subjected to carbon catabolite repression (CCR) [12-14] mediated by the transcription factor Cre1 (Carbon catabolite repressor 1) [15]. CCR is triggered by high concentrations of D-glucose and D-xylose (products of enzymatic degradation of cellulose and xylan, respectively) $[12-14,16]$. Additionally, all further described transcription factors involved in the regulation of xylanases, i.e., Ace1, Ace2, and Ace3, were reported to have also effects on the gene expression of cellulases [13, 17-20]. Thus, none of the so far known regulators can be specifically used as a target for knowledge-based strain design with the aim of shifting the cellulases-to-xylanases ratio.

In 2003, a cis-element in the xyn 2 promoter bound under repressing conditions was identified. It was described as an AGAA sequence on the non-coding strand upstream of an Xyr1 binding site [21]. A mutation of the AGAA-box led to an increased expression of a reporter gene under inducing conditions. The AGAAbox was therefore considered to be bound by a repressor. More recently, based on the development of a highly sensitive in vivo footprinting technique, an inverted repeat of the AGAA sequence further upstream was suggested to be part of the binding motif for the potential repressor [22].

Three candidate regulators binding the cis-element were identified by a pull-down assay followed by massspectrometric analysis and bioinformatic assessment [23]. The expression of the most promising regulatory protein, Xpp1 (Xylanase promoter-binding protein 1), was reported to be up-regulated in the presence of D-glucose. Additionally, a GST fusion of its DNA-binding domain as well as an in vitro translated full-length protein could bind a DNA probe containing the cis-element of the xyn 2 promoter in vitro [23].

In this study, we tested the influence of different carbon sources on the level of xpp1 expression, and deleted and over-expressed xpp 1 in $T$. reesei. To investigate the involvement of Xpp1 in the regulation of xylanase and cellulase expression in vivo, the resulting strains were compared with regard to their xylanolytic and cellulolytic activities by enzyme assays and by direct measurement of the degradation compounds. Transcript levels of the genes encoding for the main enzymes involved in the degradation of xylan and cellulose were determined. Moreover, binding of Xpp1 in vivo to its cis-element in the xyn2 promoter was investigated by in vivo footprinting.

\section{Results}

Deletion and over-expression of xpp 1 in T. reesei

To analyze the role of Xpp1 in vivo, we deleted and overexpressed $x p p 1$ in $T$. reesei. For the deletion of $x p p 1$, a gene replacement strategy by homologous recombination was applied. The structural gene of $x p p 1$ was replaced by a hygromycin resistance cassette in $T$. reese $i$ QM6a $\Delta$ tmus53. A schematic representation of the deletion strategy is shown in Additional file 1a. For overexpression of xpp 1, a co-transformation strategy was applied. The coding sequence of $x p p 1$ fused to the constitutive promoter of $p k i$ was inserted ectopically into the chromosome of T. reesei QM6a using pAN7-1, which confers hygromycin resistance [24]. The transcript levels of $x p p 1$ were measured in the obtained candidates by quantitative PCR (qPCR). A deletion strain lacking xpp 1 transcript was chosen randomly and termed QM6a $\triangle x p p 1$. The over-expression candidate exhibiting the highest relative expression of $x p p 1$ compared to its parent strain (3.5-fold over-expressed) was chosen for further experiments and termed QM6aOExpp1. Genomic modifications in both strains were confirmed by PCR and by Southern blot analysis (Additional file 1b, c). Neither the deletion strain QM6a $\triangle x p p 1$ nor the overexpression strain QM6aOExpp1 showed any differences in growth or sporulation behavior compared to their parent strains in minimal medium (liquid cultures or plates) containing lactose or carboxymethylcellulose (CMC), and surprisingly neither on xylan. On D-glucose, the deletion strain grows slightly slower compared to the parent strain (see Additional file 2).

\section{In vivo DNA binding of Xpp1 under repressing conditions}

As mentioned, Xpp1 was identified by a pull-down assay using a probe that contained the AGAA-boxes of the 
xyn 2 promoter [23]. To test whether this motif is bound in vivo by Xpp1, we performed a dimethyl sulfate (DMS)induced in vivo footprint comparing the xpp1 deletion strain and its parent strain. In a replacement experiment, both strains were pre-grown on glycerol and then transferred to minimal medium containing $50 \mathrm{mM}$ D-glucose. We could observe drastic differences between the two strains comparing the accessibility of the part of the $x y n 2$

$-241$

GGAGAACAACTTCTAGACTGGGTAA

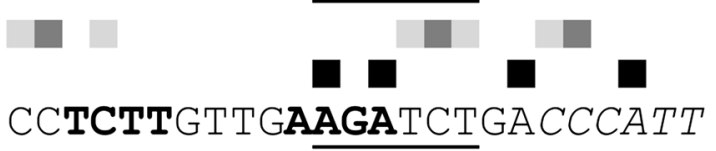

Fig. 1 In vivo footprinting analysis of the Xpp1-binding region within the xyn2 promoter. T. reesei QM6a $\Delta$ tmus 53 and the xpp 1 deletion strain were pre-cultured on glycerol and thereafter transferred to MA medium containing $50 \mathrm{mM}$ D-glucose. DNA was methylated in vivo with DMS after $3 \mathrm{~h}$ incubation. Analysis of data was performed using ivFAST [22]. Significant differences in methylation intensities of individual purine nucleotides between the two strains are represented by squares (white, no difference; light gray, difference with ratios of more than 1.1 and less than 1.3; dark gray, differences with ratios of more than 1.3 and less 1.5; black, differences with ratios of more than 1.5). The sequence of the coding (upper lane) and non-coding (lower lane) strand from positions -241 to -220 of the xyn2 promoter is given. Bold letters indicate the previously described AGAA-boxes, underlined letters the palindromic sequence, and italic letters an atypical Xyr1binding site [20]. promoter that contains the AGAA-boxes (Fig. 1). Two nucleotides in the downstream AGAA-box were strongly hypermethylated in the $x p p 1$ deletion strain, but only one nucleotide of the upstream AGAA-box. Interestingly, three nucleotides adjacent to the downstream AGAAbox were also hypermethylated. These three nucleotides are part of a palindromic sequence $\left(5^{\prime}\right.$-TCTAGA- $\left.3^{\prime}\right)$ that overlaps with the downstream AGAA-box (Fig. 1).

\section{Induction of xpp 1 gene expression}

Previously, the gene expression of $x p p 1$ was reported to be up-regulated by $\mathrm{D}$-glucose [23]. We were interested whether D-xylose has an effect on the transcript levels of xpp1. To test this, T. reesei QM6a $\Delta$ tmus53 was replaced on $50 \mathrm{mM}$ D-glucose, $0.5 \mathrm{mM}$ D-xylose, or $66 \mathrm{mM}$ $\mathrm{D}$-xylose, and transcript levels of xpp1 were measured by a qPCR assay. We found both D-glucose and high levels of $\mathrm{D}$-xylose to induce the gene expression of xpp1 (Fig. 2a). To gain further insights into the regulation of gene expression of xpp1, we additionally compared its transcript levels in a growth experiment on different carbon sources, i.e., D-glucose, D-xylose, and glycerol [1\% (w/v) each]. Samples were taken when similar amounts of biomass had accumulated on the different carbon sources. We found that transcript levels of $x p p 1$ decreased on all three carbon sources at the later time point (Fig. 2b). This finding prompted us to investigate whether this decrease was caused by the depletion of an easily utilizable carbon source or by a change in growth rate. To this end, we grew T. reesei in $1 \%(\mathrm{w} / \mathrm{v}) \mathrm{D}$-glucose for $24 \mathrm{~h}$ at $30^{\circ} \mathrm{C}$ and then

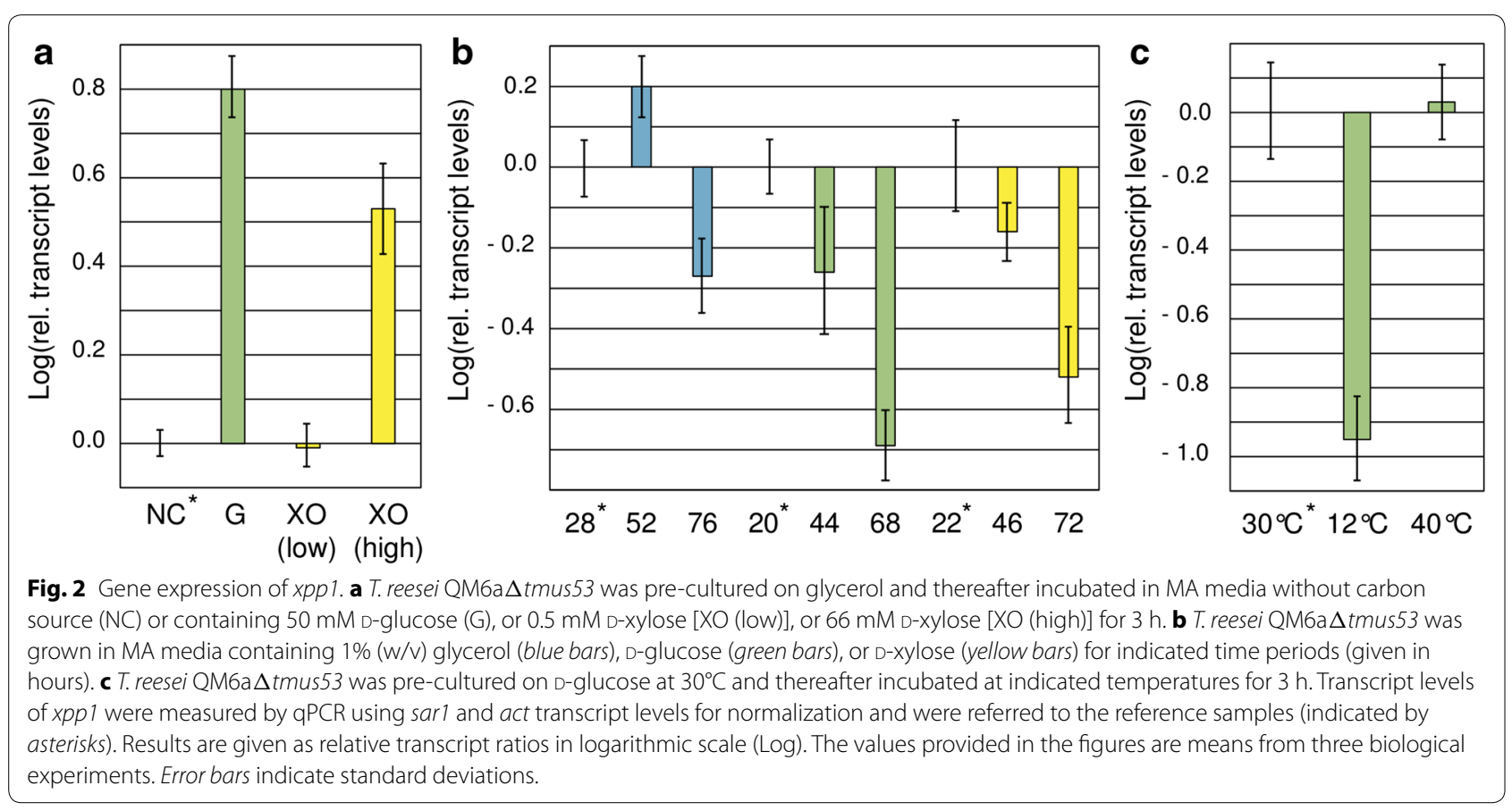


changed the temperature for $3 \mathrm{~h}$. We found the $x p p 1$ transcript levels drastically down-regulated at $12^{\circ} \mathrm{C}$ (Fig. 2c). On the other hand, a temperature shift to $40^{\circ} \mathrm{C}$ did not influence the $x p p 1$ transcript levels, indicating that eventual stress caused by the temperature change was not the reason for the observed down-regulation at $12^{\circ} \mathrm{C}$.

\section{Deletion of xpp 1 leads to higher xylanolytic activities in $T$. reesei}

To examine the influence of Xpp1-the suggested repressor of the expression of the endo-xylanase XYNII [21, 23] - on the xylanolytic phenotype of T. reesei, the xpp 1 deletion, over-expression, and parent strain were grown on xylan. We measured substantial higher endo-xylanolytic activity in the resulting culture supernatant of the deletion strain and lower endo-xylanolytic activity in the over-expression strain compared to the parent strain after $72 \mathrm{~h}$ (Fig. 3a). Additionally, we detected higher $\beta$-xylosidase activity in the culture supernatant of the deletion strain compared to its parent strain and lower activity in the over-expression strain (Fig. 3b).

We were interested whether the synergistic action of the xylanolytic enzymes differed between the deletion strain and its parent strain during cultivation on xylan. As we could not detect free oligo- or monosaccharides in the supernatant during the growth experiment, we used sterile-filtered culture supernatants of the two strains to degrade xylan in vitro. The degradation was monitored over time by measuring the concentration of free xylose oligomers and D-xylose. Xylose oligomers (xylobiose to xyloheptaose) were released at a higher initial rate in the assay using culture supernatant of the xpp1 deletion strain compared to the one of the parent strain. The concentrations of xylopentaose and xylotriose are shown as examples in Fig. 3c. This points to a higher endo-xylanolytic activity of the xpp 1 deletion strain. However, the maximal intermediate concentration of xylopentaose did not increase for xpp1 deletion, but was similar to that of the parent strain (approximately, $75 \mu \mathrm{g} / \mathrm{mL}$; see Fig. 3c). Considering the higher endo-xylanolytic activity of the xpp1 deletion strain, the observed similar concentration maxima additionally point to a higher $\beta$-xylanolytic activity because xylopentaose is primarily generated by the action of the endo-xylanases and degraded by $\beta$-xylosidase. This conclusion is supported by the faster increase of $\mathrm{D}$-xylose concentration in the assay using culture supernatant of the xpp 1 deletion strain compared to the parent strain (Fig. 3c), since D-xylose is exclusively released by the action of $\beta$-xylosidase.

\section{Xpp1 regulates xyn2 transcription at later cultivation stages}

We aimed to test whether Xpp1 does in fact act as a transcription factor on the regulation of gene expression of
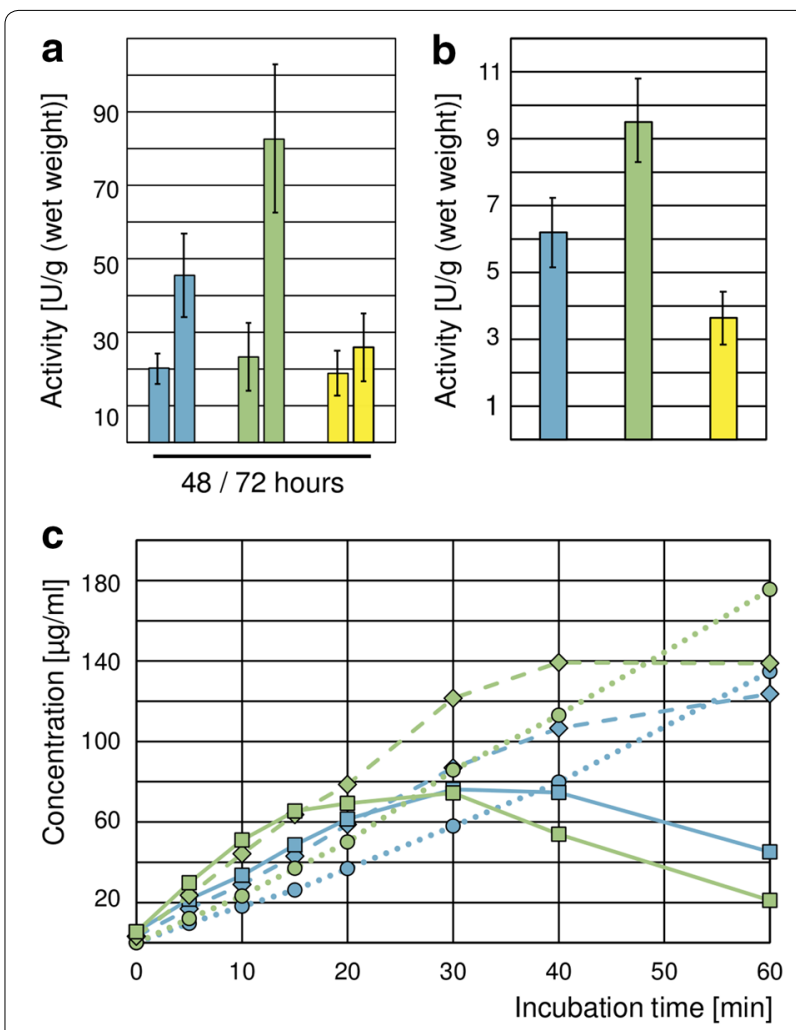

Fig. 3 Influence of Xpp1 on the xylanolytic activities of T. reesei. T. reesei QM6a $\Delta$ tmus53 (blue bars), the xpp1 deletion strain (green bars), and the xpp 1 over-expression strain (yellow bars) were grown in MA medium containing $1 \%$ xylan for 48 and $72 \mathrm{~h}$. The endo-xylanolytic activities (a) and the $\beta$-xylosidase activity (b) were measured and normalized to the acquired biomass after $72 \mathrm{~h}$. The values provided in the figures are means from three biological experiments. Error bars indicate standard deviations. $\mathbf{c}$ Xylan was degraded in vitro with culture supernatants (xylan, $72 \mathrm{~h}$ ) of T. reesei QM6a $\Delta$ tmus53 (blue) and the xpp 1 deletion strain (green) in a time course experiment. Concentrations of xylopentaose (squares, solid lines), xylotriose (diamonds, dashed lines), and D-xylose (circles, dotted lines) are given.

$x y n 2$ directly. In a first experiment, the xpp 1 deletion strain and its parent strain were subjected to a replacement experiment using $50 \mathrm{mM}$ D-glucose or $66 \mathrm{mM}$ D-xylose. The transcript levels of xyn2 were quantified by a qPCR assay. Surprisingly, we could not observe higher $x y n 2$ transcript levels in the deletion strain on both tested carbon sources after $3 \mathrm{~h}$ compared to the parent strain (Fig. 4a). Xpp1 does not seem to have an effect on the inducibility of $x y n 2$ gene expression, which conflicts with the elevated xylanolytic activity of the xpp 1 deletion strain observed, e.g., after $72 \mathrm{~h}$ growth on xylan. Therefore, we monitored xyn 2 transcript levels in the two strains throughout growth on xylan. We could detect higher xyn 2 transcript levels in the deletion strain compared to its parent strain after $30,36,48$, and $72 \mathrm{~h}$ (Fig. 4b). Only the early ( $24 \mathrm{~h}) x y n 2$ transcript level 

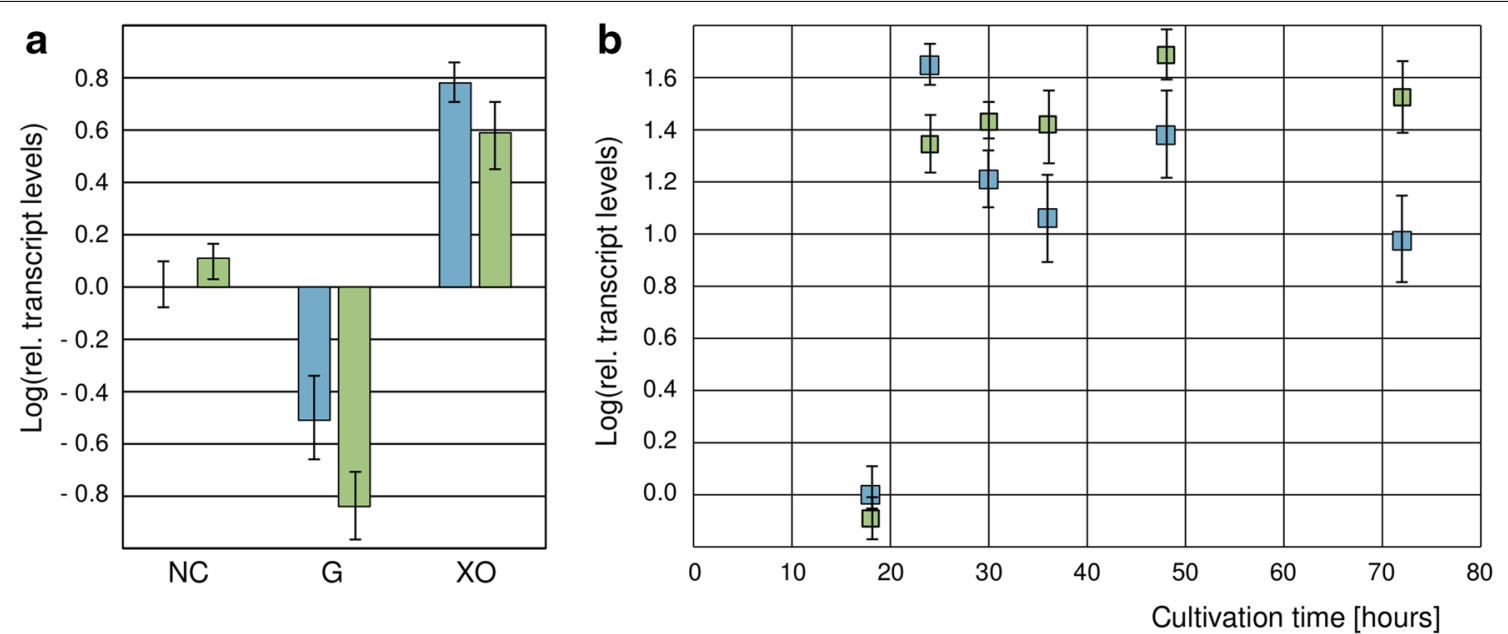

Fig. 4 Influence of Xpp1 on transcript levels of xyn2 in T. reesei. a T. reesei QM6a $\Delta$ tmus53 (blue bars) and the xpp1 deletion strain (green bars) were pre-cultured on glycerol and thereafter transferred to MA media without carbon source (NC), or containing 50 mM D-glucose (G), or 66 mM D-xylose (XO) and incubated for $3 \mathrm{~h}$. Transcript levels refer to the reference sample (QM6a $\Delta$ tmus53, NC). b T. reesei QM6a $\Delta$ tmus53 (blue squares) and the xpp 1 deletion strain (green squares) were grown in MA medium containing 1\% (w/v) xylan. Samples were taken after 18, 24, 30, 36, 48, and 72 h of cultivation. Transcript levels of xyn 2 were measured by qPCR using sar1 and act transcript levels for normalization and referred to the reference sample (QM6a $\Delta$ tmus53, 18 h). Results are given as relative transcript ratios in logarithmic scale (Log). The values provided in the figures are means from three biological experiments. Error bars indicate standard deviations.

is lower in the deletion strain. These findings match the observed xylanolytic activities. Deletion and parent strain had the same xylanolytic activities after $48 \mathrm{~h}$ (Fig. 3a). During growth $x y n 2$ transcript levels drop in the parent strain, whereas they stay at a high level in the deletion strain (Fig. 4b), which results in a higher xylanolytic activity of the deletion strain at later cultivation stages (Fig. 3a).

\section{Xpp 1 controls the gene expression of $x y n 1$ and the putative $b \times 12$}

Since further enzymes besides XYNII contribute to the xylanolytic activities of $T$. reesei, we investigated if Xpp1 also controls their expression. First, we analyzed xyn1 transcript levels, which turned out to be higher in the $x p p 1$ deletion strain compared to the parent strain (Fig. 5a), similar to $x y n 2$ (compare Fig. 4b). Since we detected increased $\beta$-xylosidase activity in the supernatant of the xpp1 deletion strain (compare Fig. 3b), we measured the $b x l 1$ transcript levels. Surprisingly, we detected equal levels in both strains (Fig. 5b), which contradicts the measured enzyme activity. However, the protein ID 58450 (on http://genome.jgi-psf.org/Trire2/ Trire2.home.html) is annotated as a putative $\beta$-xylosidase [25]. Consequently, we analyzed the transcript levels of the gene encoding for this candidate $\beta$-xylosidase (in the following termed $b x l 2$ ). They were indeed higher in the xpp1 deletion strain compared to its parental strain at later cultivation stages (Fig. 5c).

\section{Xpp1 does not affect the initial step of $D-x y l o s e$ metabolism}

Degradation of xylan results in the release of D-xylose, which is taken up by $T$. reesei and metabolized via the pentose phosphate way. The initial reaction is the reduction of $\mathrm{D}$-xylose to xylitol, catalyzed by xylose reductase (EC1.1.1.307). We measured xylose reductase activity of the xpp 1 deletion strain and its parent strain after $72 \mathrm{~h}$ growth on xylan. We could not detect any difference between the two strains (Fig. 6a). Further, the transcript levels of $x y l 1$, encoding the xylose reductase, were monitored in the two strains throughout growth on xylan. Samples were taken periodically and $x y l 1$ transcript levels were measured with a qPCR assay. Matching the obtained enzymatic activities, no differences of $x y l 1$ transcript levels could be observed between the two strains (Fig. 6b). Xpp1 regulates the gene expression of the extracellular xylanolytic enzymes, but not of the intracellular xylose reductase. We presume that the xylose reductase can be used as an indicator for the whole downstream D-xylose metabolism in this context.

\section{Cellulase expression is not regulated by Xpp1}

Owing to the fact that cellulolytic and xylanolytic enzymes are co-regulated in $T$. reesei, we were interested to know to what extent Xpp1 influences the expression of cellulases. The xpp1 deletion strain, the over-expression strain, and the parent strain were cultivated in minimal medium 


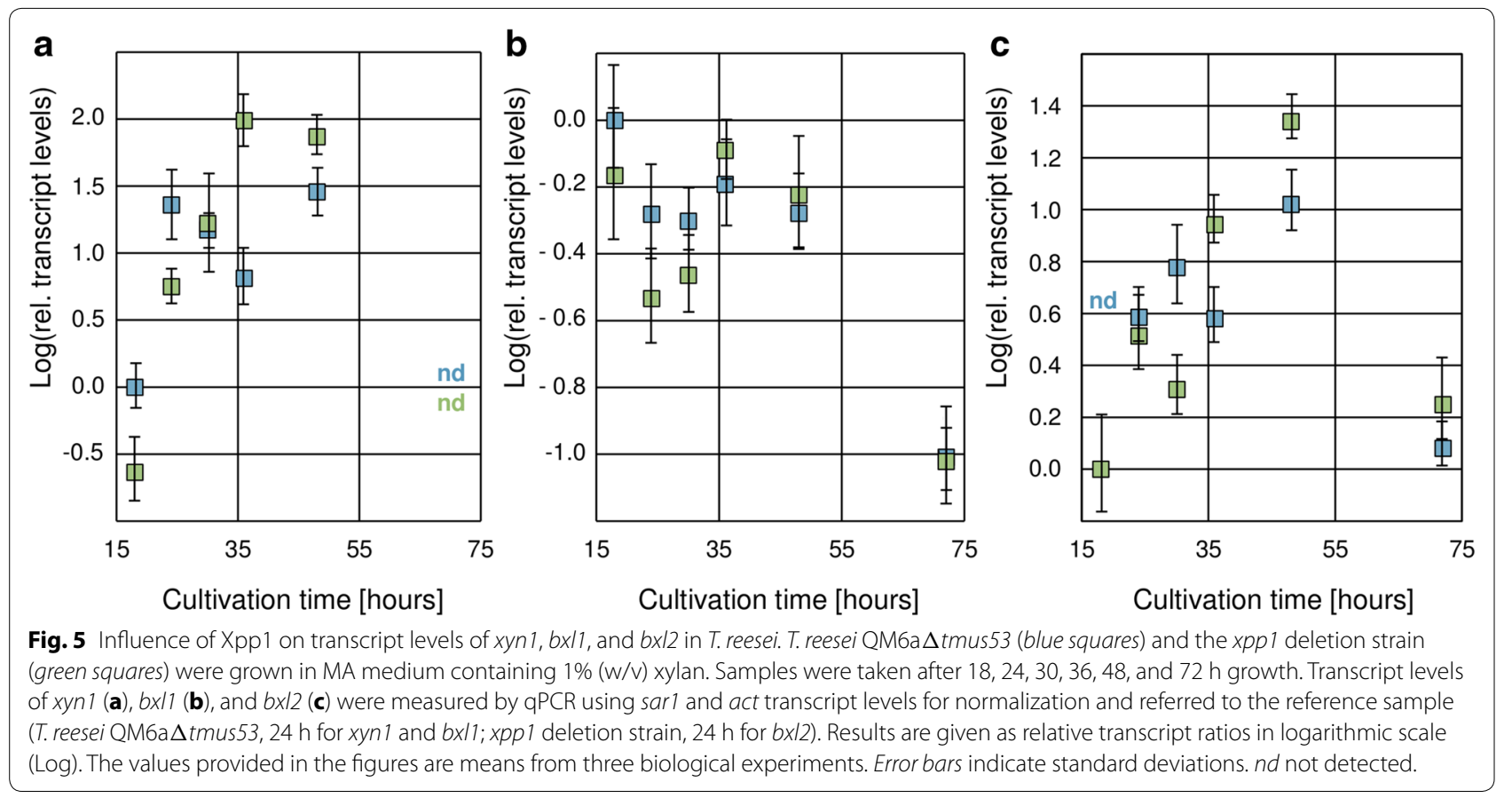

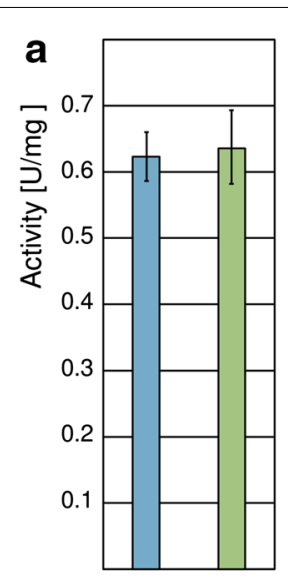

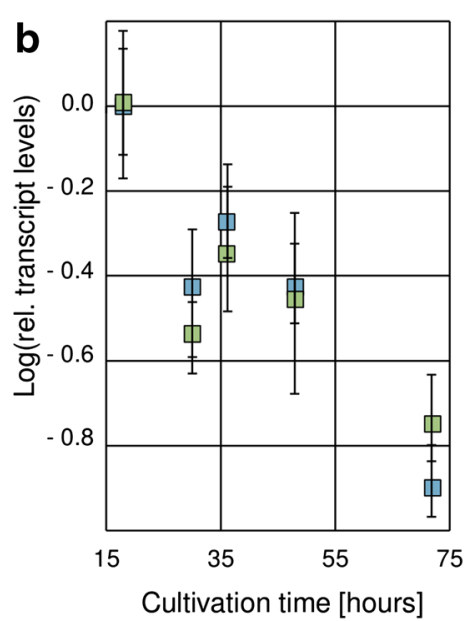

Fig. 6 Influence of Xpp1 on xylose reductase expression in T. reesei. T. reesei QM6a $\Delta$ tmus53 (blue) and the xpp1 deletion strain (green) were grown in MA medium containing $1 \%(\mathrm{~W} / \mathrm{v})$ xylan. Samples were taken after $18,30,36,48$, and $72 \mathrm{~h}$ growth. a Xylose reductase activity in cell-free extracts was measured in vitro and normalized to the total protein concentration of the cell-free extracts. b Transcript levels of the $x y / 1$ gene were measured by qPCR using sar 1 and act transcript levels for normalization and were referred to the reference sample (T. reesei QM6a $\Delta$ tmus53, $18 \mathrm{~h}$ ). Results are given as relative transcript ratios in logarithmic scale (Log). The values provided in the figures are means from three biological experiments. Error bars indicate standard deviations.

containing CMC for $72 \mathrm{~h}$, and endo-cellulolytic activities of the resulting culture supernatants were assayed. We could not detect any differences on comparing the three strains (Fig. 7a). Further, transcript levels of the main cellulases, i.e., $\operatorname{cbh} 1, \operatorname{cbh} 2$, and egl1, were monitored throughout growth in CMC with qPCR assays. No differences between the deletion strain and the parent strain could be observed (Fig. 7b, c, data for cbh2 not shown).

\section{Discussion}

Two inverted AGAA-boxes in the xyn2 promoter were previously considered to be the binding motif of a repressor [21, 22]. Xpp1 was later identified as the most promising candidate repressor [23]. Using in vivo footprinting, we could detect strong differences at the putative binding site of Xpp1 in the xyn 2 promoter comparing the $x p p 1$ deletion strain and its parent strain. However, Xpp1 is a basic helix-loop-helix protein containing a predicted E-box binding motif [23]. The classical E-box is a hexameric palindrome with the consensus $5^{\prime}$-CANNTG-3' [26]. Interestingly, the AGAA-box on the non-coding strand within the $x y n 2$ promoter overlaps with a hexameric palindrome (5'-TCTAGA-3'). Five of the six nucleotides of this palindromic sequence were hypermethylated during in vivo footprinting in the absence of Xpp1. A similar hexameric palindrome $\left(5^{\prime}\right.$-ACTAGT-3') can be found in the promoters of $x y n 1$ and $b x l 2$. Notably, both promoters contain also an inverted AGAA-repeat. In summary, we observed a correlation between regulation by Xpp1 and the presence of a hexameric palindrome $5^{\prime}$-WCTAGW-3' together with an inverted AGAA-repeat. Table 1 provides an overview on the genes investigated during this study as potential targets of Xpp1 regulation and the presence of relevant cis elements in their promoters. 

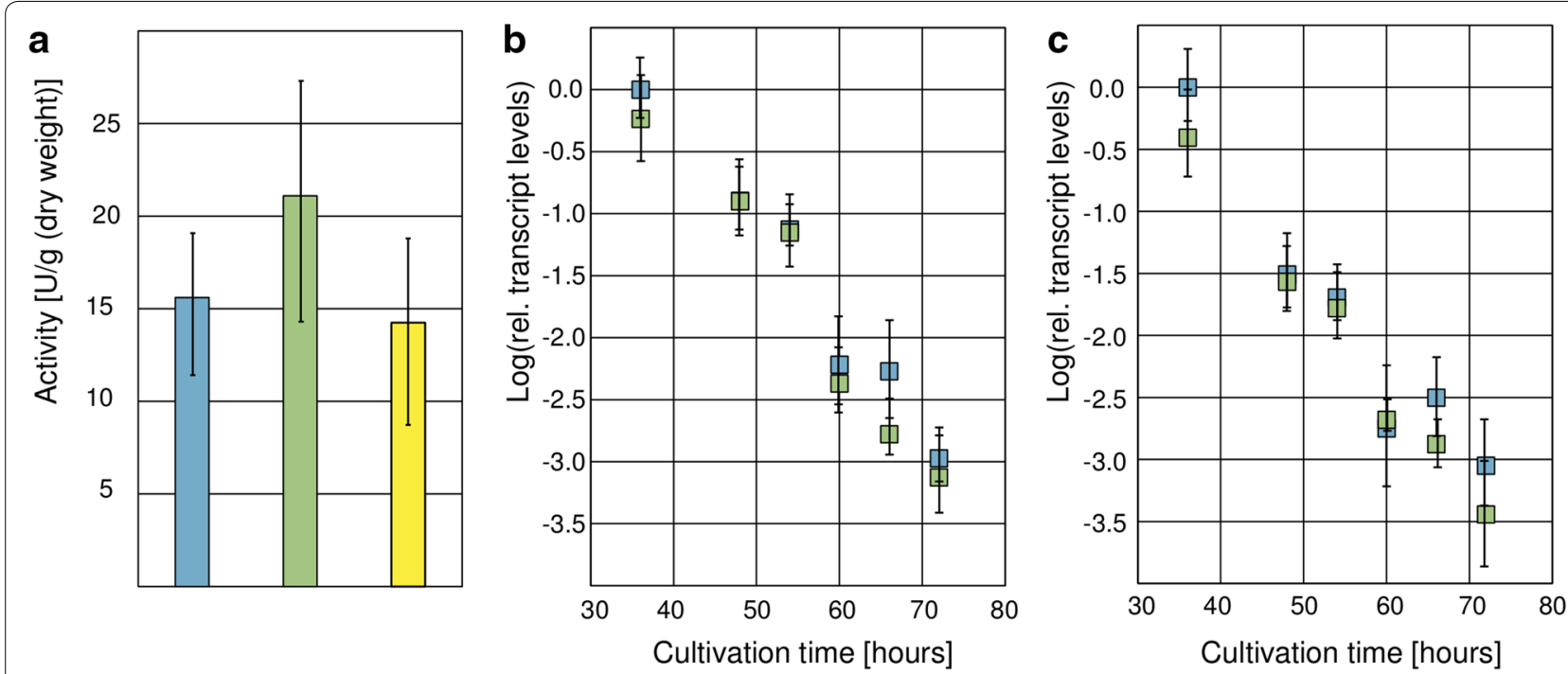

Fig. 7 Influence of Xpp1 on cellulase expression in T. reesei. T. reesei QM6a $\Delta$ tmus53 (blue), the xpp1 deletion strain (green), and the xpp1 over-expression strain (yellow) were grown in MA medium containing $1 \%(\mathrm{w} / \mathrm{v}) \mathrm{CMC}$ for 36, 48, 54, 60, 66, and $72 \mathrm{~h}$. a Endo-cellulolytic activity was measured and normalized to the acquired biomass after $72 \mathrm{~h}$. Relative transcript levels of $c b h 1$ (b) and eg/1 (c) were measured by qPCR using sarl and act transcript levels for normalization and were referred to the reference sample (T. reesei QM6a $\Delta$ tmus $53,36 \mathrm{~h}$ ). Results are given as relative transcript ratios in logarithmic scale (Log). The values provided in the figures are means from three biological experiments. Error bars indicate standard deviations.

Table 1 Overview on investigated genes concerning the Xpp1 regulation

\begin{tabular}{|c|c|c|c|}
\hline Gene & $\begin{array}{l}\text { Regulated } \\
\text { by Xpp1 }\end{array}$ & $\begin{array}{l}\text { Inverted } \\
\text { AGAA repeat }^{a}\end{array}$ & $\begin{array}{l}\text { WCTAGW } \\
\text { palindrom }^{2}\end{array}$ \\
\hline xyn2 & $x$ & $x$ & $x$ \\
\hline xyn1 & $x$ & $x$ & $x$ \\
\hline$b \times 11$ & - & - & - \\
\hline$b \times 12$ & $x$ & $x$ & $x$ \\
\hline$x y / 1$ & - & - & - \\
\hline cbh1 & - & - & - \\
\hline cbh2 & - & $x$ & - \\
\hline egll & - & - & - \\
\hline xyr1 & - & - & - \\
\hline
\end{tabular}

a Present in the promoter within $1 \mathrm{kbp}$ upstream of the start codon.

Further, an atypical Xyr1 binding site [20] downstream of the palindromic sequence within the $x y n 2$ promoter was also hypermethylated in the in vivo footprint assay in the xpp1 deletion strain, notably, to a lesser extent than the palindromic motif. However, we could not observe a difference of xyr1 transcript levels comparing deletion and parent strain during growth on xylan (unpublished observations by Derntl C, Mach RL, Mach-Aigner AR). The observed differences might be the mere result of a changed accessibility of the whole region due to the absence of Xpp1.

Deletion of $x p p 1$ resulted in elevated $x y n 1, x y n 2$, and $b x l 2$ transcript levels at later cultivation stages in the deletion strain and in higher endo-xylanolytic and $\beta$-xylosidase activities in the supernatant after $72 \mathrm{~h}$, but not after $48 \mathrm{~h}$. Xpp1 seems to act as a repressor of gene expression of xylanolytic enzymes at later time points. Transcript levels of $x y n 2, x y n 1$, and $b x l 2$ are higher in the deletion strain compared to the parent strain at late cultivation stages. We speculate that these observed differences are the result of a CCR-independent feedback mechanism mediated by Xpp1. During this study, we observed that high concentrations of $\mathrm{D}$-xylose upregulate Xpp1 expression, which acts as repressor on the expression of xylanolytic enzymes. Considering that accumulation of high amounts of D-xylose is a relatively slow process, this would fit the general idea of a feedback mechanism. This model would also explain why Xpp1 does not influence the inducibility of xyn2 expression at early time points (compare Fig. 4a, b). According to our hypothesis, the role of Xpp1 is the down-regulation of an induced system, but not the prevention of induction. The nature of this feedback mechanism might also explain the observed equal growth behavior of parent, deletion, and over-expression strains. All strains have similar initial xylanolytic activities and therefore provide similar amounts of monosaccharides for the growing fungi in the beginning. Biomass formation occurs during this period, resulting in similar growth behaviors.

Remarkably, Xpp1 regulates only the xylanolytic part of the lignocellulose-degrading enzymes in T. reesei. Transcript levels of the main cellulases as well as cellulolytic activities were not changed in the deletion and the overexpression strain compared to the parental strain. As 
mentioned earlier, regulation of gene expression of the main cellulases seems to be regulated in a direct manner by the amount of available Xyr1 [11]. Matching the obtained results, we could not observe a difference of xyr1 transcript levels comparing deletion and parent strain throughout growth on CMC (unpublished observations by Derntl C, Mach RL, Mach-Aigner AR).

\section{Conclusions}

Xpp1 seems to be the mediator of a feedback mechanism regulating gene expression of xylanases. Xpp1 does not influence transcription of the main cellulolytic enzymes. We believe that this makes Xpp1 a highly attractive candidate for knowledge-based strain design, as this regulatory protein is a very promising instrument for shifting the cellulases-to-xylanases ratio in the secreted protein fraction during industrial cellulase production.

\section{Methods}

\section{Fungal strains}

T. reesei strains QM6a (ATCC 13631), QM6a $\Delta$ tmus53 [27], the xpp1 deletion strain QM6a $\Delta$ tmus $53 \Delta x p p 1$ (QM6a $\triangle x p p 1$, this study), and the $x p p 1$ over-expression strain (QM6aOExpp1, this study) were maintained on malt extract (MEX) agar at $30^{\circ} \mathrm{C}$. Hygromycin $\mathrm{B}$ was added when applicable to a final concentration of $113 \mathrm{U} / \mathrm{mL}$.

\section{Growth conditions}

For carbon source replacement experiments, strains were pre-cultured in 1-L Erlenmeyer flasks on a rotary shaker $(180 \mathrm{rpm})$ at $30^{\circ} \mathrm{C}$ for $22 \mathrm{~h}$ in $300 \mathrm{~mL}$ of Mandels-Andreotti (MA) medium [28] containing 1\% (w/v) glycerol as the sole carbon source. A total of $10^{9}$ conidia per liter (final concentration) was used as the inoculum. Pre-grown mycelia were washed, then equal amounts were resuspended in MA media containing D-xylose and $\mathrm{D}$-glucose in final concentrations as given or in medium without carbon source (reference condition), and harvested after $3 \mathrm{~h}$ of incubation.

For direct cultivation on D-glucose, D-xylose, glycerol, or xylan (Lenzing AG, Lenzing, Austria) strains were grown in 500-mL Erlenmeyer flasks in a rotary shaker $(180 \mathrm{rpm})$ at $30^{\circ} \mathrm{C}$ in $100 \mathrm{~mL}$ of MA medium containing $1 \%(\mathrm{w} / \mathrm{v})$ of the respective carbon source. For direct cultivation on $\mathrm{CMC}$, strains were grown in 1-L Erlenmeyer flasks stationary at $30^{\circ} \mathrm{C}$ in $60 \mathrm{~mL}$ of MA medium containing 1\% (w/v) CMC (Carl Roth GmbH, Karlsruhe, Germany). Mycelium and supernatant were separated by filtration through Miracloth (EMD Millipore, part of Merck KGaA, Darmstadt, Germany). Mycelia grown on xylan were weighed directly as reference for enzymatic assays. Mycelia grown on $\mathrm{CMC}$ were dried at $80^{\circ} \mathrm{C}$ overnight prior to weighing.

\section{Plasmid construction}

Escherichia coli strain Top10 (Invitrogen, part of Life Technologies, Paisley, UK) was used for all cloning purposes throughout this study and maintained on LB at $37^{\circ} \mathrm{C}$. Ampicillin and hygromycin $\mathrm{B}$ were added when applicable to final concentrations of $100 \mathrm{mg} / \mathrm{mL}$ and 113 $\mathrm{U} / \mathrm{mL}$, respectively.

PCRs for all cloning purposes were performed with Phusion High-Fidelity DNA Polymerase (Thermo Scientific, Waltham, MA, USA) according to the manufacturer's instructions. All used primers are listed in Additional file 3. Generation of competent E. coli cells and subsequent transformation was performed according to standard protocols using $\mathrm{CaCl}_{2}$.

For the construction of $\mathrm{pCD}-\Delta \mathrm{xpp} 1$, the $5^{\prime}$-flank of xpp 1 was amplified by PCR using chromosomal DNA of T. reesei QM6a as template with the primers xpp1-5fwDNotI and xpp1-5rev. The PCR product was inserted into an EcoRV-digested pJET1.2 (Thermo Scientific) in the opposite direction of Eco47IR (killer gene), yielding pJET$5^{\prime}$-xpp1. The $3^{\prime}$-flank of $x p p 1$ was amplified by PCR using chromosomal DNA of $T$. reesei QM6a as template with the primers xpp1-3fwd and xpp1-3rev-NotI. This PCR product was inserted into pJET-5'-xpp1 in the same direction as the $5^{\prime}$-flank. For this purpose, the plasmid was digested with ClaI and blunted with T4 DNA polymerase. The resulting plasmid was termed pJET-BFxpp1. Finally, a hygromycin resistance cassette was generated by PCR using pRLM $\mathrm{EX} 30$ [29] as template with the primers Ppki_5fwd and Tcbh2_rev-Bcul and inserted into pJET-BFxpp1 which was previously digested with NcoI and blunted with T4 DNA polymerase. The orientation was determined by sequencing and found to be the same as the $5^{\prime}$-flank and the $3^{\prime}$-flank of xpp 1 .

For construction of $\mathrm{pCD}_{\mathrm{ex}} \mathrm{xpp} 1$, the coding sequence of $x p p 1$ was amplified by PCR using cDNA from $T$. reesei QM6a as template with the primers xpp1_fwD-XbaI and xpp1_rev-NsiI. The PCR product was digested with $\mathrm{XbaI}$ and NsiI and ligated into $\mathrm{pRLM}_{\mathrm{EX}} 30$ [29] digested accordingly.

\section{Fungal protoplast transformation}

Protoplast transformation of $T$. reesei was performed as described by Gruber et al. [30]. For deletion of xpp1, NotI-digested pCD- $\Delta$ xpp1 was used for transformation of T. reesei QM6a $\Delta$ tmus53. For over-expression of xpp1 under the control of the constitutive promoter of $p k i$, $\mathrm{pCD}_{\mathrm{ex}} \mathrm{xpp} 1$ was co-transformed together with pAN7-1 [24] into T. reesei QM6a. The transformation reaction was added to $80 \mathrm{~mL}$ melted, $50^{\circ} \mathrm{C}$ warm MEX agar containing 1.2 M sorbitol. This mixture was poured into four sterile Petri dishes and incubated at $30^{\circ} \mathrm{C}$ for $2-5$ days until colonies were visible. The resulting candidates were 
subjected to four rounds of homokaryon selection by streaking.

\section{Isolation of chromosomal DNA and PCR analysis}

Chromosomal DNA was isolated from mycelium by grinding in liquid nitrogen followed by a phenol/chloroform extraction. RNA was degraded using RNaseA (Thermo Scientific). DNA was precipitated with isopropanol, washed with $70 \%(\mathrm{v} / \mathrm{v})$ ethanol, and dissolved in $\mathrm{ddH}_{2} \mathrm{O}$. For PCR analysis, $10 \mathrm{ng}$ of chromosomal DNA was used as template in a $25-\mu \mathrm{L}$ PCR using GoTaq $^{\circledR}$ G2 polymerase (Promega, Madison, WI, USA) according to the manufacturer's instructions. All used primers are listed in Additional file 3. For subsequent agarose gel electrophoresis of DNA fragments, a GeneRuler $1 \mathrm{~kb}$ DNA Ladder (Thermo Scientific) was applied for estimation of fragment size. DNA sequencing was performed at Microsynth (Balgach, Switzerland).

\section{Southern blot analysis}

$15 \mu \mathrm{g}$ of chromosomal DNA were digested with $30 \mathrm{U}$ of the given restriction enzymes. The resulting DNA fragments were separated by electrophoresis on a $0.8 \%$ agarose gel, then denatured in $0.4 \mathrm{M} \mathrm{NaOH}$, and transferred by capillary forces onto a Biodyne B $0.45 \mu \mathrm{m}$ nylon membrane (Pall Corporation, Port Washington, NY, USA) using $10 \times$ SSC. $1.5 \mu \mathrm{g}$ of biotinylated DNA probe were used for hybridization at $65^{\circ} \mathrm{C}$ overnight. Labeling of the probe was performed using a Klenow Fragment (exo-) (Thermo Scientific), random hexamer primers, and biotin-11-dUTP (Jena Bioscience, Jena, Germany). Signals were visualized by using Poly-HRP conjugated to streptavidin and ECL Plus Western Blotting substrate (both Thermo Scientific Pierce, part of Life Technologies) on a ChemiDoc MP (Bio-Rad Laboratories, Hercules, USA).

\section{RNA extraction and reverse transcription}

0.01-0.03 g of harvested mycelia were homogenized in $1 \mathrm{~mL}$ of peqGOLD TriFast DNA/RNA/protein purification system reagent (PEQLAB Biotechnologie, Erlangen, Germany) using a FastPrep FP120 BIO101 ThermoSavant cell disrupter (Qbiogene, Carlsbad, USA). RNA was isolated according to the manufacturer's instructions, and the concentration was measured using the NanoDrop 1000 (Thermo Scientific).

Synthesis of cDNA from mRNA was carried out using the RevertAid ${ }^{\mathrm{TM}} \mathrm{H}$ Minus First Strand cDNA Synthesis Kit (Thermo Scientific) according to the manufacturer's instructions.

\section{Transcript analysis}

Quantitative PCRs were performed in a Mastercycler ${ }^{\circledR}$ ep realplex 2.2 system (Eppendorf, Hamburg, Germany). All reactions were performed in triplicate. The amplification mixture (final volume $25 \mu \mathrm{L}$ ) contained $12.5 \mu \mathrm{L} 2 \times \mathrm{iQ}$ SYBR Green Mix (Bio-Rad Laboratories, Hercules, USA), $100 \mathrm{nM}$ forward and reverse primer, and $2.5 \mu \mathrm{L}$ cDNA (diluted 1:100). Primer sequences are provided in Additional file 3. Cycling conditions and control reactions were performed as described previously [31]. Calculations using sar 1 and act 1 as reference genes were performed as published previously [31].

\section{In vivo footprinting}

In vivo methylation of DNA using DMS followed by ligation-mediated PCR was performed as described previously [22] using the primers RG127, RG128, RG129, RG130, RG131, and RG132 for investigation of a regulatory region bearing the AGAA-box within the xyn2 promoter. Primer sequences are provided in Additional file 3. FAM-labeled fragments were analyzed by capillary gel electrophoresis (Microsynth) and results were analyzed using the program ivFAST [22].

\section{Determination of enzymatic activities}

Endo-xylanolytic and endo-cellulolytic activities of cultivation supernatants were measured with Xylazyme AX tablet assay and Cellazyme $\mathrm{C}$ tablet assay (both Megazyme International Ireland, Wicklow, Ireland) according to the manufacturer's instructions, respectively. One unit of activity is defined as the amount of enzyme required to release $1 \mu$ mole of reducing-sugar equivalents per minute.

The $\beta$-xylosidase activity was determined with the substrate 4-nitrophenyl- $\beta$-D-xylopyranoside (Sigma-Aldrich Corporation, St. Louis, MO, USA) as described previously [32]. One unit of activity is defined as the amount of enzyme required to release $1 \mu \mathrm{mol}$ of $\mathrm{D}$-xylose reducing-sugar equivalents per minute.

For the measurement of xylose reductase activity, mycelia were ground in liquid nitrogen with mortar and pestle. $100 \mathrm{mg}$ was resuspended in $1 \mathrm{~mL} 0.1 \mathrm{M}$ Tris/ $\mathrm{HCl}$ $\mathrm{pH} 7.5,1 \mathrm{mM}$ EDTA, and $5 \mathrm{mM} \beta$-mercaptoethanol and incubated at $4{ }^{\circ} \mathrm{C}$ for $15 \mathrm{~min}$. Cell debris was removed by centrifugation at $20,000 \mathrm{~g}$ at $4{ }^{\circ} \mathrm{C}$ for $10 \mathrm{~min}$. The protein concentration of the resulting cell extract was determined photometrically by measuring the absorption at $280 \mathrm{~nm}$ using NanoDrop 1000 (Thermo Scientific). $200 \mu \mathrm{L}$ of the cell extract was added to a $2 \mathrm{~mL}$ reaction in $0.1 \mathrm{M}$ Tris, pH 7.5, containing $10 \mathrm{mM}$ D-xylose and $0.15 \mathrm{mM}$ $\mathrm{NADPH}$. Oxidation of NADPH to NAD+ was monitored photometrically at $340 \mathrm{~nm}$ in a Jasco V-360 spectrophotometer (Jasco Corporation, Hachioji City, Japan) for $1 \mathrm{~min}$ at $25^{\circ} \mathrm{C}$. One unit is defined as the amount that causes a reduction of optical density at $340 \mathrm{~nm}$ of 0.01 per minute. The activity was normalized to the total protein concentration of the cell extract. 
High-performance anion exchange chromatography with pulsed amperometric detection (HPAEC-PAD) analysis For monitoring xylan degradation in vitro, $1 \mathrm{~mL}$ sterilefiltered cultivation supernatant from $72 \mathrm{~h}$ growth in MA medium containing $1 \%$ xylan (Lenzing AG) was mixed with $9 \mathrm{~mL}$ sterile MA medium containing 1\% xylan (Lenzing AG) and incubated in a $50 \mathrm{~mL}$ sterile reaction tube on a rotary shaker $(180 \mathrm{rpm})$ at $30^{\circ} \mathrm{C}$. $200 \mu \mathrm{L}$ samples were drawn at given time points and the enzymatic reactions immediately stopped by addition of $200 \mu \mathrm{L} 100 \mathrm{mM}$ $\mathrm{NaOH}$. The remaining, undissolved xylan was removed by centrifugation at $4^{\circ} \mathrm{C}$ and $20,000 \mathrm{~g}$ for $10 \mathrm{~min}$. Samples were diluted 1:10 with $10 \mathrm{mM} \mathrm{NaOH}$ for HPAEC-PAD analysis. HPAEC-PAD analysis was performed with a Thermo Scientific Dionex ICS-5000 system (Thermo Scientific). Samples were separated on a Dionex CarboPac PA1 $(2 \times 250 \mathrm{~mm})$ carbohydrate column (Thermo Scientific). The column was preconditioned at $0.26 \mathrm{~mL} / \mathrm{min}$ in $150 \mathrm{mM} \mathrm{NaOH} / 250 \mathrm{mM} \mathrm{NaOAc}$ for $5 \mathrm{~min}$, followed by $150 \mathrm{mM} \mathrm{NaOH}$ for $15 \mathrm{~min}$. Samples were then injected and oligosaccharides separated by a linear gradient to $150 \mathrm{mM} \mathrm{NaOH} / 250 \mathrm{mM} \mathrm{NaOAc}$ in $20 \mathrm{~min}$. Quantification was done by calibration of peak areas with authentic standards of xylooligosaccharides (Megazyme International, Ireland).

\section{Additional files}

Additional file 1: Deletion and over-expression of xpp 1 (A) Deletion of xpp 1 in the parent strain QM6a $\Delta$ tmus53 ( $\Delta$ tmus53) by homologous recombination with the plasmid $\mathrm{pCD}-\Delta \mathrm{xpp} 1$ yielding an $x p p 1$ deletion strain ( $\Delta$ tmus53 $\Delta x p p 1$ ) is represented schematically. Position of the xpp 1 locus on scaffold 16 is indicated at the top. Thin black arrows indicate the approximate positions of the primers used for genotype analysis via PCR (5f2, xpp1-5fwd2 and Pkr, Ppki_5rev). Black-rimmed, light gray arrow represents the xpp 1 gene; hatched boxes represent regions for homologous recombination; thick, black arrow represents the hph gene; gray arrow indicate the homologous recombination event; gray, dotted lines represent genomic DNA sequence; solid, black lines represent plasmid DNA sequence. (B) Correct and exclusive integration of the hygromycin cassette in the xpp 1 locus was verified by Southern Blot analysis. The obtained signals correspond to the expected fragment sizes after digestion with Pstl (3,410 bp and 4,622 bp) using the hph coding region as probe. (C) Ectopic insertions of xpp 1 expression cassettes in the over-expression strain were determined by Southern Blot analysis using the xpp 1 coding region as probe. L, 1-kb DNA ladder; $P$, parent strain (QM6a $\Delta$ tmus53); $\Delta, x p p 1$ deletion strain; O, xpp 1 over-expression strain.

Additional file 2: Impact of Xpp1 on growth of T. reesei. (A) T. reesei QM6a $\Delta$ tmus53 (blue) and the xpp1 deletion strain (green) were grown in MA medium containing $1 \%$ (w/v) D-glucose (squares, solid lines) or lactose (triangles, dashed lines), or CMC (circles, dotted lines) for 18, 24, 30,38 , and 50 hours. The values provided in the figures are means from three biological experiments. Error bars indicate standard deviations. (B) T. reesei QM6a $\Delta$ tmus53 (left lane) and the xpp1 deletion strain (right lane) were pre-grown on MA medium plates containing glycerol. Equal pieces of overgrown agar were transferred to MA medium plates containing $1 \%(\mathrm{~W} / \mathrm{V})$ D-glucose $(\mathrm{G})$ or xylan $(\mathrm{XN})$ or lactose $(\mathrm{L})$ or CMC. Pictures were taken after 48 hours growth at $30^{\circ} \mathrm{C}$ in darkness.

Additional file 3: Primers used throughout this study.

\section{Abbreviations}

Ace1/2/3: Activator of cellulases 1/2/3; CCR: carbon catabolite repression CMC: carboxymethylcellulose; Cre1: Carbon catabolite repressor 1; DMS: dimethyl sulfate; EDTA: ethylenediaminetetraacetic acid; FAM: fluorescein amidite; GST: glutathione S-transferase; HPAEC-PAD: high-performance anion exchange chromatography with pulsed amperometric detection; ivFAST: in vivo footprinting analysis software tool; MA: Mandels-Andreotti; MEX: malt extract; qPCR: quantitative PCR; Xpp1: Xylanase promoter-binding protein 1; Xyr 1: Xylanase regulator 1 .

\section{Authors' contributions}

$\mathrm{CD}$ constructed the plasmids and the deletion and over-expression strains; performed the growth experiments, the qPCR assays, and the enzymatic assays; and drafted this manuscript. AR performed the in vivo footprinting assays. ES performed the HPAEC-PAD analysis. RLM participated in the conception of the study and revised the manuscript. ARMA participated in the conception of the study, supervised the experiments, and revised the manuscript. All authors read and approved the final manuscript.

\section{Author details}

${ }^{1}$ Department for Biotechnology and Microbiology, Institute of Chemical Engineering, TU Wien, Gumpendorfer Str. 1a, 1060 Vienna, Austria. ${ }^{2}$ Department of Biochemical Engineering, Institute of Chemical Engineering, TU Wien, Gumpendorfer Str. 1a, 1060 Vienna, Austria.

\section{Acknowledgements}

This work was supported by two grants from the Austrian Science Fund (FWF), P24851 and V232 given to ARMA, and by a doctoral program ("AB-Tec") of Vienna University of Technology. We thank Prof. A. Friedl, Vienna University of Technology, for providing access to the HPAEC equipment.

\section{Compliance with ethical guidelines}

Competing interests

The authors declare that they have no competing interests.

Received: 4 March 2015 Accepted: 24 July 2015

Published online: 06 August 2015

\section{References}

1. Kuhls K, Lieckfeldt E, Samuels GJ, Kovacs W, Meyer W, Petrini O et al (1996) Molecular evidence that the asexual industrial fungus Trichoderma reesei is a clonal derivative of the ascomycete Hypocrea jecorina. Proc Natl Acad Sci USA 93:7755-7760

2. Cherry JR, Fidantsef AL (2003) Directed evolution of industrial enzymes: an update. Curr Opin Biotechnol 14:438-443

3. Nidetzky B, Claeyssens M (1994) Specific quantification of Trichoderma reesei cellulases in reconstituted mixtures and its application to cellulasecellulose binding studies. Biotechnol Bioeng 44:961-966

4. Tolan J, Foody B (1999) Cellulase from submerged fermentation. In: Tsao GT, Brainard AP, Bungay HR, Cao NJ, Cen P, Chen Z, Du J, Foody B, Gong CS, Hall P, Ho NWY, Irwin DC, Iyer P, Jeffries TW, Ladisch CM, Ladisch MR, Lee YY, Mosier NS, Mühlemann HM, Sedlak M, Shi N-Q, Tsao GT, Tolan JS, Torget RW, Wilson DB, Xia L (eds) Recent progress in bioconversion of lignocellulosics, vol 65. Springer Berlin, Heidelberg, pp 41-67 (Advances in Biochemical Engineering/Biotechnology)

5. Kubicek CP, Harman GE, Ondik KL (eds) (1998) Trichoderma and gliocladium. Taylor \& Francis, Bristol

6. Kumar R, Singh S, Singh OV (2008) Bioconversion of lignocellulosic biomass: biochemical and molecular perspectives. J Ind Microbiol Biotechnol 35:377-391

7. Törrönen A, Harkki A, Rouvinen J (1994) Three-dimensional structure of endo-1,4-beta-xylanase II from Trichoderma reesei: two conformational states in the active site. EMBO J 13:2493-2501

8. Margolles-Clark E, Tenkanen M, Nakari-Setälä T, Penttilä M (1996) Cloning of genes encoding alpha-L-arabinofuranosidase and beta-xylosidase from Trichoderma reesei by expression in Saccharomyces cerevisiae. Appl Environ Microbiol 62:3840-3846 
9. Ek M, Gellerstedt G, Henriksson G (2009) Pulp and Paper Chemistry and Technology. Volume 1, Wood Chemistry and Wood Biotechnology. Walter de Gruyter, Berlin

10. Stricker AR, Grosstessner-Hain K, Wurleitner E, Mach RL (2006) Xyr1 (xylanase regulator 1 ) regulates both the hydrolytic enzyme system and D-xylose metabolism in Hypocrea jecorina. Eukaryot Cell 5:2128-2137

11. Derntl C, Gudynaite-Savitch L, Calixte S, White T, Mach RL, Mach-Aigner AR (2013) Mutation of the Xylanase regulator 1 causes a glucose blind hydrolase expressing phenotype in industrially used Trichoderma strains. Biotechnol Biofuels 6:62

12. IImén M, Saloheimo A, Onnela ML, Penttilä ME (1997) Regulation of cellulase gene expression in the filamentous fungus Trichoderma reesei. Appl Environ Microbiol 63:1298-1306

13. Rauscher R, Würleitner E, Wacenovsky C, Aro N, Stricker AR, Zeilinger S et al (2006) Transcriptional regulation of xyn 1, encoding xylanase I, in Hypocrea jecorina. Eukaryot Cell 5:447-456

14. Mach-Aigner AR, Pucher ME, Steiger MG, Bauer GE, Preis SJ, Mach RL (2008) Transcriptional regulation of $x y r 1$, encoding the main regulator of the xylanolytic and cellulolytic enzyme system in Hypocrea jecorina. Appl Environ Microbiol 74:6554-6562

15. Strauss J, Mach RL, Zeilinger S, Hartler G, Stöffler G, Wolschek M et al (1995) Cre1, the carbon catabolite repressor protein from Trichoderma reesei. FEBS Lett 376:103-107

16. Mach-Aigner AR, Pucher ME, Mach RL (2010) D-Xylose as a repressor or inducer of xylanase expression in Hypocrea jecorina (Trichoderma reesei). Appl Environ Microbiol 76:1770-1776

17. Aro N, Saloheimo A, Ilmén M, Penttilä M (2001) ACEll, a novel transcriptional activator involved in regulation of cellulase and xylanase genes of Trichoderma reesei. J Biol Chem 276:24309-24314

18. Aro N, Ilmen M, Saloheimo A, Penttila M (2003) ACEl of Trichoderma reesei is a repressor of cellulase and xylanase expression. Appl Environ Microbiol 69:56-65

19. Häkkinen M, Valkonen MJ, Westerholm-Parvinen A, Aro N, Arvas M, Vitikainen $M$ et al (2014) Screening of candidate regulators for cellulase and hemicellulase production in Trichoderma reesei and identification of a factor essential for cellulase production. Biotechnol Biofuels 7:14

20. Stricker AR, Trefflinger P, Aro N, Penttilä M, Mach RL (2008) Role of Ace2 (activator of cellulases 2) within the xyn2 transcriptosome of Hypocrea jecorina. Fungal Genet Biol FGB 45:436-445

21. Würleitner E, Pera L, Wacenovsky C, Cziferszky A, Zeilinger S, Kubicek CP et al (2003) Transcriptional regulation of xyn2 in Hypocrea jecorina. Eukaryot Cell 2:150-158
22. Gorsche R, Jovanovic B, Gudynaite-Savitch L, Mach RL, Mach-Aigner AR (2014) A highly sensitive in vivo footprinting technique for conditiondependent identification of cis elements. Nucleic Acids Res 42:e1

23. Mach-Aigner AR, Grosstessner-Hain K, Poças-Fonseca MJ, Mechtler K, Mach RL (2010) From an electrophoretic mobility shift assay to isolated transcription factors: a fast genomic-proteomic approach. BMC Genom $11: 644$

24. Punt PJ, Oliver RP, Dingemanse MA, Pouwels PH, van den Hondel CAMJJ (1987) Transformation of Aspergillus based on the hygromycin B resistance marker from Escherichia coli. Gene 56:117-124

25. Martinez D, Berka RM, Henrissat B, Saloheimo M, Arvas M, Baker SE et al (2008) Genome sequencing and analysis of the biomass-degrading fungus Trichoderma reesei (syn. Hypocrea jecorina). Nat Biotechnol 26:553-560

26. Chaudhary J, Skinner MK (1999) Basic helix-loop-helix proteins can act at the E-box within the serum response element of the c-fos promoter to influence hormone-induced promoter activation in Sertoli cells. Mol Endocrinol Baltim Md 13:774-786

27. Steiger MG, Vitikainen M, Uskonen P, Brunner K, Adam G, Pakula T et al (2011) Transformation system for Hypocrea jecorina (Trichoderma reesei) that favors homologous integration and employs reusable bidirectionally selectable markers. Appl Environ Microbiol 77:114-121

28. Mandels M (1985) Applications of cellulases. Biochem Soc Trans 13:414-416

29. Mach RL, Schindler M, Kubicek CP (1994) Transformation of Trichoderma reesei based on hygromycin $B$ resistance using homologous expression signals. Curr Genet 25:567-570

30. Gruber F, Visser J, Kubicek CP, de Graaff LH (1990) The development of a heterologous transformation system for the cellulolytic fungus Trichoderma reesei based on a pyrG-negative mutant strain. Curr Genet 18:71-76

31. Steiger MG, Mach RL, Mach-Aigner AR (2010) An accurate normalization strategy for RT-qPCR in Hypocrea jecorina (Trichoderma reesei). J Biotechnol 145:30-37

32. Kristufek D, Zeilinger S, Kubicek CP (1995) Regulation of $\beta$-xylosidase formation by xylose in Trichoderma reesei. Appl Microbiol Biotechno 42:713-717

\section{Submit your next manuscript to BioMed Central and take full advantage of:}

- Convenient online submission

- Thorough peer review

- No space constraints or color figure charges

- Immediate publication on acceptance

- Inclusion in PubMed, CAS, Scopus and Google Scholar

- Research which is freely available for redistribution

Submit your manuscript at

www.biomedcentral.com/submit

C Biomed Central 